\title{
Caractère pseudo-périodique des fluctuations longitudinales de vitesse dans les écoulements turbulents
}

\author{
par J. Larras \\ Inspecteur Gènéral des Ponts et Chaussées
}

\section{Introduction}

I

L'expérience montre que les fluctuations longitudinales de vitesse d'un écoulement turbulent relèvent des lois du hasard, mais que les plus rapides d'entre elles présentent un certain caractère de périodicité, du même genre que celui des dunes de sable ou des vagues de la mer.

Or, E. L. Deacon a relevé des périodes moyennes de fluctuation des vitesses d'un écoulement d'air de l'ordre de quelques secondes à l'aide d'un anémomètre dont il évaluait le pouvoir de résolution à 2 secondes $\left({ }^{1}\right), R$. H. Sherlock a relevé des périodes moyennes de l'ordre de la fraction de seconde à l'aide d'un anémomètre dont il évaluait le pouvoir de résolution à 0,25 ou 0,5 secondes $\left({ }^{2}\right), \mathrm{S}$. Pond, $\mathrm{R}$. W. Stexart et R.W. Burling ont relevé des périodes moyennes de l'ordre de $1 / 80^{\circ}$ de seconde à l'aide d'un anémomètre à fil chaud dont ils évaluaient le pouvoir de résolution à $1 / 100^{\mathrm{e}} \mathrm{de}$ seconde $\left({ }^{3}\right)$, et J. P. Millat a relevé des périodes moyennes de fluctuation des vitesses d'un écoulement d'eau de l'ordre de quelques dix-millièmes de seconde à l'aide d'appareil à fil chaud dont il évaluait le pouvoir de résolution à $1 / 10000^{\mathrm{e}}$ de seconde (figure 1) $\left({ }^{4}\right)$.

On en est donc conduit à se demander s'il n'existerait pas un lien direct, de cause à effet, entre le pouvoir de résolution de l'appareillage que l'on utilise pour mesurer les vitesses instantanées d'écoulement d'un fluide et la pseudo-

(1) Gust variation with height up to $150 \mathrm{~m}$. Quarterly Journal of the Royal Meteorological Society, vol 81, 1955.

(2) Variation of wind velocity and gusts with height. Proceedings of the American Society of Civil Engineers, vol 78, 1952 (separate $\mathrm{n}^{\circ} 126$ ).

(3) Turbulence spectra in the wind over wawes. Journal of the atmospheric sciences, vol $20, \mathrm{n}^{\circ} 4,1963$.

(4) Etude experimentale de l'écoulement turbulent dans un divergent bidimensionnel parcouru par de l'air. Publications scientifiques et techniques du Ministère de l'Air. Paris, 1957, $\mathrm{n}^{\circ} 335$ périodicité des fluctuations de vitesse que l'on relève au sein de cet écoulement.

Nous nous proposons de montrer dans la présente étude que c'est bien ce qui se passe effectivement dans la réalité.

II

L'inertie (mécanique, calorifique, électrique,...) de l'appareillage de mesure ne permet de relever les valeurs instantanées des vitesses qu'avec une certaine distorsion, et l'on ne peut relever par suite que la moyenne mobile d'un certain nombre de ces valeurs.

Nous allons montrer que ce "lissage " d'origine instrumentale provoque l'apparition de festons pseudopériodiques le long des enregistrements de vitesse en fonction du temps, tant pour une répartition gaussienne que pour une répartition uniforme des fluctuations aléatoires de la variable en fonction du temps. Mais nous ne saurions affirmer, pour autant, que l'existence de festons plus ou moins périodiques le long des enregistrements «lissés» constitue la preuve a contrario du caractère purement aléatoire des fluctuations de vitesse correspondantes en fonction du temps.

Les phénomènes de "lissage " des fluctuations purement aléatoires d'une variable relèvent au premier chef de la théorie statistique des mesures développées par S.O. Rice dans le Bell System Technical Journal de juillet 1944 et

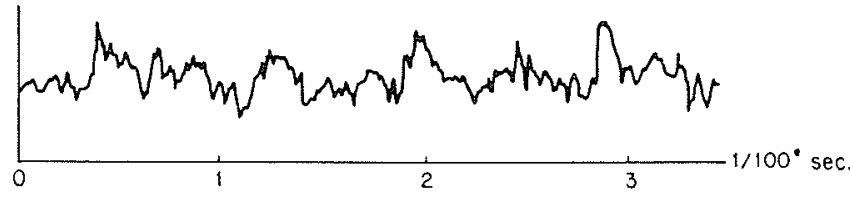

( D'après J.P. Milliat)

1/ Fluctuations longitudinales de vitesse (Vitesse moyenne $1,49 \mathrm{~m} / \mathrm{s}$ ) 
janvier 1945, mais c'est une théorie dont les applications pratiques et, même, la simple lecture nécessitent de profondes connaissances mathématiques, et nous lui avons préféré, pour la vulgarisation de la méthode comme pour la commodité de l'exposé, l'emploi d'une méthode, moins générale mais extrêmement simple, de simulation numérique.

\section{Simulations numériques utilisées}

Nous avons simulé une distribution uniforme des fluctuations de vitesse en fonction du temps en utilisant la suite des décimales du nombre $\pi$, dont nous avons remplacé toutefois les zéros par des 10 (car l'introduction de la valeur 0 équivaudrait à supprimer systématiquement l'un des 10 nombres de la répartition dans le calcul des moyennes mobiles). Les 500 et 1000 premières décimales de $\pi$ se répartissent, en effet, comme suit :

\begin{tabular}{rrr}
\hline & 500 & 1000 \\
\hline 1 & $11,6 \%$ & $11,5 \%$ \\
2 & $10,8 \%$ & $10,3 \%$ \\
3 & $10,0 \%$ & $10,2 \%$ \\
4 & $10,6 \%$ & $9,3 \%$ \\
5 & $10,0 \%$ & $9,7 \%$ \\
6 & $9,6 \%$ & $9,4 \%$ \\
7 & $7,2 \%$ & $9,5 \%$ \\
8 & $10,6 \%$ & $10,1 \%$ \\
9 & $10,4 \%$ & $10,6 \%$ \\
10 & $9,2 \%$ & $9,6 \%$ \\
\hline
\end{tabular}

Nous avons recoupé, d'autre part, les résultats obtenus à partir de cette série de décimales en utilisant la suite des 480 entiers allant de 0 à 19 , et répartis au hasard, dont M. G. Kendall et A. Stuart ont donné le tableau à la page 380 du volume 3 de leur ouvrage sur « The advanced theory of statistics ». Ces nombres se répartissent, d'une façon un peu moins uniforme, comme suit :

\begin{tabular}{lllll}
\hline 0 & $3,7 \%$ & - & 10 & $4,4 \%$ \\
1 & $5,2 \%$ & - & 11 & $5,0 \%$ \\
2 & $5,6 \%$ & - & 12 & $4,2 \%$ \\
3 & $5,8 \%$ & - & 13 & $6,0 \%$ \\
4 & $5,2 \%$ & - & 14 & $5,2 \%$ \\
5 & $5,4 \%$ & - & 15 & $7,1 \%$ \\
6 & $4,6 \%$ & - & 16 & $3,9 \%$ \\
7 & $5,0 \%$ & - & 17 & $5,4 \%$ \\
8 & $5,6 \%$ & - & 18 & $4,6 \%$ \\
9 & $4,8 \%$ & - & 19 & $3,3 \%$ \\
\hline
\end{tabular}

II

Nous avons simulé une distribution gaussienne des fluctuations de vitesse en fonction du temps en procédant à 500 tirages au sort successifs sur des bulletins que nous avions numérotés de 1 à 9 dans les proportions suivantes :

$\begin{array}{lllllllllll}\text { Entiers ...... } & 1 & 2 & 3 & 4 & 5 & 6 & 7 & 8 & 9\end{array}$

$\begin{array}{llllllllll}\text { Proportions.. } & 2 & 5 & 13 & 22 & 26 & 22 & 13 & 5 & 2\end{array}$

Cette simulation ne diffère, en effet, qu'extrêmement peu d'une loi de Gauss, comme il ressort des proportions relevées à l'issue des 500 tirages au sort précédents :

\begin{tabular}{crrr}
\hline $\begin{array}{c}\text { ENTIERS } \\
\text { INFERIEURS } \\
\text { OU ÉGAUX A }\end{array}$ & $\begin{array}{c}\text { Proportions } \\
\text { THÉORIQUES }\end{array}$ & Gauss & $\begin{array}{c}\text { TiRAGE AU } \\
\text { SORT }\end{array}$ \\
\hline 1 & $1,8 \%$ & $1,8 \%$ & $1,4 \%$ \\
2 & $6,4 \%$ & $6,5 \%$ & $5,4 \%$ \\
3 & $18,2 \%$ & $18,4 \%$ & $16,6 \%$ \\
4 & $38,2 \%$ & $38,2 \%$ & $37,0 \%$ \\
5 & $61,8 \%$ & $61,8 \%$ & $61,0 \%$ \\
6 & $81,8 \%$ & $81,6 \%$ & $81,4 \%$ \\
7 & $93,6 \%$ & $93,5 \%$ & $93,0 \%$ \\
8 & $98,2 \%$ & $98,2 \%$ & $97,6 \%$ \\
9 & $100,0 \%$ & $100,0 \%$ & $100,0 \%$ \\
\hline
\end{tabular}

Nous avons recoupé, d'autre part, les résultats obtenus à partir de cette première série de bulletins en procédant à 500 nouveaux tirages au sort sur une deuxième série de bulletins que nous avions numérotés de 1 à 17 dans les proportions suivantes :

\begin{tabular}{cc|cc}
\hline ENTIERS & Proportions & EnTIERS & Proportions \\
\hline $1-.17$ & 1 & $6-12$ & 8 \\
$2-16$ & 1 & $7-11$ & 10 \\
$3-15$ & 2 & $8-10$ & 12 \\
$4-14$ & 3 & 9 & 13 \\
$5-13$ & 5 & & \\
\hline
\end{tabular}

Cette simulation nous a fourni la distribution gaussienne $\mathrm{n}^{\circ} 2$ ci-après :

\begin{tabular}{|c|c|c|c|}
\hline $\begin{array}{c}\text { ENTIERS } \\
\text { INFÉRIEURS } \\
\text { OU ÉGAUX A }\end{array}$ & $\begin{array}{l}\text { PROPORTIONS } \\
\text { THÉORIQUES }\end{array}$ & Gauss & $\begin{array}{c}\text { TIRAGE AU } \\
\text { SORT }\end{array}$ \\
\hline 1 & $1,0 \%$ & $0,9 \%$ & $0,6 \%$ \\
\hline 2 & $2,0 \%$ & $1,9 \%$ & $2,4 \%$ \\
\hline 3 & $4,1 \%$ & $4,1 \%$ & $5,0 \%$ \\
\hline 4 & $7,2 \%$ & $7,7 \%$ & $8,6 \%$ \\
\hline 5 & $12,4 \%$ & $13,3 \%$ & $14,8 \%$ \\
\hline 6 & $20,6 \%$ & $21,5 \%$ & $24,2 \%$ \\
\hline 7 & $30,9 \%$ & $31,7 \%$ & $35,2 \%$ \\
\hline 8 & $43,3 \%$ & $44,7 \%$ & $46,8 \%$ \\
\hline 9 & $56,7 \%$ & $55,3 \%$ & $58,4 \%$ \\
\hline 10 & $69,1 \%$ & $68,3 \%$ & $66,2 \%$ \\
\hline 11 & $79,4 \%$ & $78,5 \%$ & $78,4 \%$ \\
\hline 12 & $87,6 \%$ & $86,7 \%$ & $87,0 \%$ \\
\hline 13 & $92,8 \%$ & $92,3 \%$ & $93,4 \%$ \\
\hline 14 & $95,9 \%$ & $95,9 \%$ & $96,2 \%$ \\
\hline 15 & $98,0 \%$ & $98,1 \%$ & $98,0 \%$ \\
\hline 16 & $99,0 \%$ & $99,1 \%$ & $99,4 \%$ \\
\hline 17 & $100,0 \%$ & $100,0 \%$ & $100,0 \%$ \\
\hline
\end{tabular}




\section{Distribution uniforme des fluctuations de vitesse en fonction du temps}

I

Nous avons constaté par calcul numérique direct (tableau I) que l'intervalle de temps moyen $\Delta t$ entre les maximums successifs des fluctuations de vitesse "lissées" ne dépendait pratiquement :

- ni du nombre total $T$ de nombres de la suite que nous utilisions pour la simulation,

- ni du nombre $N$ d'entiers distincts qui figuraient dans cette suite.

- ni du "pas de lissage " utilisé, c'est-à-dire du nombre $L$ d'entiers dont nous établissions les moyennes mobiles successives.

Voici d'ailleurs, pour plus de précision, les résultats obtenus :

$\begin{array}{ccc}\text { Pas de lissage } & \text { Décimales de } \pi & \text { Kendall et Stuart } \\ (T=500, N=10) & (T=480, N=20)\end{array}$

$\begin{array}{rrr}L= & \Delta t=4,28 & \Delta t=4,39 \\ 17 & 4,20 & 4,03 \\ 23 & 3.95 & 4,74 \\ 29 & 3,93 & 4,29\end{array}$

$(T=1000, N=10)$

$L=10 \quad \Delta t=4,40$

L'écart quadratique moyen $\varepsilon$ sur la valeur $\Delta t$ de l'intervalle entre maximums successifs ne dépendait pratiquement pas, lui non plus, de $L$, de $N$ et de $T$, comme il résulte des chiffres suivants :

\begin{tabular}{lcc}
\hline Pas de lissage & Décimales De $\pi$ & Kendall ET StuarT \\
& $(T=500, N=10)$ & $(T=480, N=20)$
\end{tabular}

$\begin{array}{rrrr}L=10 & \varepsilon=2,10(49 \%) & \varepsilon=2,31(53 \%) \\ 17 & 2,01(48 \%) & 1,94(48 \%) \\ 23 & 1,74(44 \%) & 2,66(56 \%) \\ 29 & 1,81(46 \%) & 2,60(61 \%)\end{array}$

$$
L=10 \quad \varepsilon=2,21(50 \%)
$$

\section{II}

C'est évidemment vrai pour n'importe quelle vitesse moyenne d'écoulement. Mais ce l'est également pour n'importe quel type d'appareillage dont la loi de réponse ne permette de mesurer que les puissances $1 / 2,3 / 2$ ou 2 des vitesses instantanées d'écoulement du fluide au lieu des vitesses elles-mêmes à la puissance 1. C'est ainsi que l'on trouve :

- pour les 225 premiers nombres de Kendall et Stuart :

\begin{tabular}{crr}
\hline Puissances & $(T=225, N=20, L=17)$ \\
\hline 1 & $\Delta t=3,89$ & $\varepsilon=1,52(38 \%)$ \\
$3 / 2$ & 3,85 & $1,60(42 \%)$ \\
2 & 3,85 & $1,59(41 \%)$ \\
\hline
\end{tabular}

- pour une répartition des 500 premières décimales de $\pi$ de part et d'autre d'une moyenne de $(1+10): 2=5,5$ sans remplacer les 0 par des 10 :
PUISSANCES

$$
(T=500, N=10, L=10)
$$

$\begin{array}{crr}1 / 2 & \Delta t=4,30 & \varepsilon=2,08(48 \%) \\ 1 & 4,28 & 2,10(49 \%) \\ 3 / 2 & 4,26 & 2,15(50 \%) \\ 2 & 4,40 & 2,11(48 \%)\end{array}$

- pour une répartition des 500 premières décimales de $\pi$ de part et d'autre d'une moyenne de $(10+19): 2=14.5$ sans remplacer les 0 par des 10 :

\begin{tabular}{crr}
\hline Puissances & $(T=500, N=10, L=10)$ \\
\hline 1 & $\Delta t=4,28$ & $\varepsilon=2,10(49 \%)$ \\
4,25 & $1,90(45 \%)$ \\
\hline
\end{tabular}

- et pour une répartition des 500 premières décimales de $\pi$ de part et d'autre d'une moyenne de $(100+109)$ : $2=104,5$ sans remplacer les 0 par les 10 :
PuISSANCES
$(T=500, N=10, L=10)$ 


\section{Distribution gaussienne \\ des fluctuations de vitesse \\ en fonction du temps}

I

Nous avons constaté, par calcul numérique direct (tableau II), que l'intervalle de temps moyen $\Delta t$ entre les maximums successifs des fluctuations de vitesse «lissées » et l'écart quadratique moyen correspondant $\varepsilon$ ne dépendaient, là aussi, pratiquement pas de $L$, de $N$ et de $T$ lorsque l'appareillage utilisé comportait une loi de réponse linéaire. Et celà tout aussi bien pour la distribution gaussienne $\cdot n^{\circ} 1$ que pour la distribution gaussienne $\mathrm{n}^{\circ} 2$, malgré des valeurs très différentes de $N$ dans les deux cas :

\begin{tabular}{rrr}
\hline \multicolumn{3}{c}{$(T=500, N=9)$} \\
\hline$L=3$ & $\Delta t=4,43$ & $\varepsilon=2,34(53 \%)$ \\
10 & 4,54 & $2,79(60 \%)$ \\
17 & 4,33 & $2,18(51 \%)$ \\
23 & 4,39 & $2,18(50 \%)$ \\
29 & 4,50 & $2,45(54 \%)$ \\
\hline
\end{tabular}

$$
(T=500, N=17)
$$

$\begin{array}{rrr}3 & \Delta t=3,92 & \varepsilon=2,12(54 \%) \\ 10 & 4,24 & 1,93(46 \%) \\ 17 & 4,39 & 2,43(55 \%) \\ 23 & 4,28 & 2,46(57 \%) \\ 29 & 4,75 & 2,92(61 \%)\end{array}$

\section{II}

C'est, en outre, tout aussi vrai pour n'importe quel type d'appareillage dont la loi de réponse ne permette de mesurer que les puissances $1 / 2,3 / 2$ ou 2 des vitesses instantanées d'écoulement du fluide au lieu des vitesses elles-mêmes à la puissance 1. C'est ainsi qu'on trouve :

- pour la distribution gaussienne $n^{\circ} 1$ :

\begin{tabular}{crr}
\hline Puissances & $(T=500, N=9, L=10)$ \\
\hline & & \\
$1 / 2$ & $\Delta t=4,54$ & $\varepsilon=2,79(60 \%)$ \\
1 & 4,54 & $2,79(60 \%)$ \\
2 & 4,41 & $2,66(60 \%)$ \\
& 4,54 & $2,79(60 \%)$ \\
\hline
\end{tabular}

- pour la distribution gaussienne $n^{0} 2$ :

\begin{tabular}{crr}
\hline Puissances & $(T=500, N=17, L=10)$ \\
\hline & $\Delta t=4,24$ & $\varepsilon=1,93(46 \%)$ \\
1 & 4,24 & $1,93(46 \%)$ \\
2 & & \\
\hline
\end{tabular}

- et pour les distributions gaussiennes obtenues en échelonnant les valeurs instantanées des fluctuations de vitesse du fluide de la distribution gaussienne $\mathrm{n}^{\circ} 1$ de 11 à 19 , ou de 101 à 109 , au lieu de 1 à 9 avant de la « lisser » :

\begin{tabular}{crr}
\hline Puissances & $(T=500, N=9, L=10)$ \\
\hline & $\Delta t=4,54$ & $\varepsilon=2,79(60 \%)$ \\
4,54 & $2,79(60 \%)$ \\
2 & & \\
\hline
\end{tabular}

\section{III}

Tous ces résultats sont extrêmement voisins de ceux que nous avons indiqués plus haut pour des fluctuations uniformes de vitesse, tout au moins pour les valeurs de $T$ de l'ordre de 480 à 500 que nous avons utilisées ici. La «période " des fluctuations de vitesse du fluide en fonction du temps ne dépend pratiquement que du pouvoir de résolution de l'appareillage de mesure utilisé :

\begin{tabular}{lcc}
\hline & M moy. & $\varepsilon$ moy. \\
\hline & & \\
Répartitions gaussiennes $\ldots$. & 4,44 & 2,51 \\
Répartitions uniformes $\ldots . .$. & 4,25 & 2,11 \\
& & \\
\hline
\end{tabular}

et ne dépend pratiquement pas de la loi de réponse suivant laquelle cet appareillage convertit les quantités observées en résultats d'observation.

\section{Amplitude des fluctuations pseudo-périodiques de vitesse en fonction du temps}

Nous représenterons l'amplitude des fluctuations pseudo-périodiques des vitesses d'écoulement du fluide en fonction du temps par la différence, pour chaque maximum de vitesse, entre ce maximum lui-même et la moyenne arithmétique des 2 minimums qui l'encadrent.

Pour les distributions uniformes des fluctuations de vitesse, l'amplitude moyenne $\Delta v$ est plus ou moins inverse- 
ment proportionnelle au " pas de lissage " $L$ (ou pouvoir de résolution) de l'appareillage de mesure, et d'autant plus élevée que la densité de répartition des variables (représentée $\operatorname{par} N$ ) est plus grande :

\begin{tabular}{rrr}
\hline & $\begin{array}{c}\text { Décimales De } \pi \\
(T=500, N=10)\end{array}$ & $\begin{array}{c}\text { Kendall ET STUART } \\
(T=480, N=20)\end{array}$ \\
\hline & & \\
10 & $L \Delta v=7,91$ & $L \Delta v=12,87$ \\
17 & 6,93 & 13,34 \\
23 & 6,06 & 11,19 \\
29 & 6,83 & 13,08 \\
\hline
\end{tabular}

Il en est de même pour les distributions gaussiennes :

\begin{tabular}{rrr}
\hline & $\begin{array}{c}\text { Distribution No } \\
(T=500, N=9)\end{array}$ & $\begin{array}{c}\text { Distribution No } 2 \\
(T=500, N=17)\end{array}$ \\
\hline & & \\
& & \\
& & \\
10 & $\Delta v=4,10$ & \\
17 & 4,06 & 7,34 \\
23 & 4,02 & 8,03 \\
29 & 4,09 & 8,18 \\
& 4,09 & 11,29 \\
\hline
\end{tabular}

II

L'amplitude moyenne des fluctuations pseudo-périodiques de vitesse dépend beaucoup de la loi de réponse suivant laquelle l'appareillage de mesure convertit les quantités observées en résultats d'observation, et ceci tout aussi bien pour les distributions uniformes des fluctuations de vitesse :

\begin{tabular}{|c|c|c|}
\hline Puissances & $\begin{array}{l}\text { DÉCIMALES DE } \pi \\
\quad(T=500) \\
(N=10, L=10)\end{array}$ & $\begin{array}{c}\text { KENDALL ET STUART } \\
(T=225) \\
(N=20, L=\operatorname{L} 17)\end{array}$ \\
\hline 1 & $L \Delta v=7,91$ & $L \Delta v=14,20$ \\
\hline $3 / 2$ & 24,07 & 56,01 \\
\hline 2 & 77,72 & 233,88 \\
\hline
\end{tabular}

que pour les distributions gaussiennes:

\begin{tabular}{ccc}
\hline Puissances & $\begin{array}{c}\text { Distribution No } 1 \\
(T=500) \\
(N=9, L=10)\end{array}$ & $\begin{array}{c}\text { Distribution } N^{\circ} 2 \\
(T=500) \\
(N=17, L=10)\end{array}$ \\
\hline 1 & $L \Delta v=4,06$ & $L \Delta v=\begin{array}{c}7,37 \\
13,18\end{array}$ \\
2 & 41,84 & 133,63 \\
\hline
\end{tabular}

\section{III}

L'amplitude moyenne des fluctuations pseudo-périodiques de vitesse en fonction du temps est de l'ordre de 10 à $20 \%$ de la moyenne générale $V$ des vitesses instantanées que l'on observerait en l'absence de « lissage » pour n'importe quelle loi de réponse de l'appareillage de mesure. Du moins pour les distributions uniformes des fluctuations de vitesse :

\begin{tabular}{crr}
\hline Puissances & $\begin{array}{c}\text { Décimales } D E \pi \\
(T=500)\end{array}$ & $\begin{array}{c}\text { Kendall Et Stuart } \\
(T=225)\end{array}$ \\
& $(N=10, L=10)$ & $(N=20, L=17)$ \\
\hline & & \\
$3 / 2$ & $\Delta v / V=14,4 \%$ & $\Delta v / V=15,0 \%$ \\
2 & $17,4 \%$ & $9,8 \%$ \\
& $20,2 \%$ & $10,9 \%$ \\
\hline
\end{tabular}

car l'amplitude moyenne des fluctuations pseudopériodiques en fonction du temps est généralement plus faible pour les distributions gaussiennes que pour les distributions uniformes.

\section{Dérive pseudo-périodique}

\section{I}

Les fluctuations pseudo-périodiques de vitesse dont nous venons de parler s'accompagnent de phénomènes de dérive qui se traduisent par des ondulations de vitesse beaucoup plus amples et plus lentes, dont l'amplitude maximale $A$ (c'est-à-dire la différence entre extremums) dépend du «pas de lissage » $L$ comme suit :

\begin{tabular}{ccc}
\hline Pas de lissage & Décimales De $\pi$ & Kendall et Stuart \\
& $(N=10, T=500)$ & $(N=20, T=480)$
\end{tabular}

$\begin{array}{rrr}L=10 & A L=40 & A L=106 \\ 17 & 51 & 132 \\ 23 & 52 & 155 \\ 29 & 64 & 178\end{array}$


L'amplitude $A$ des grandes ondulations lentes en fonction du temps est de l'ordre d'une dizaine de fois l'amplitude $\Delta v$ des petites fluctuations pseudo-périodiques de vitesse et, par suite, de l'ordre de la moyenne générale $V$ des vitesses instantanées que l'on observerait en l'absence de " lissage » (figure 1) :

\begin{tabular}{|c|c|c|}
\hline PAS DE LISSAGE & $\begin{array}{l}\text { DÉCIMALES DE } \pi \\
(N=10, T=500)\end{array}$ & $\begin{array}{l}\text { KENDALL ET STUART } \\
(N=20, T=480)\end{array}$ \\
\hline $\begin{array}{r}L=10 \\
17 \\
23 \\
29\end{array}$ & $\begin{aligned} A / \Delta v= & 5,05 \\
& 7,35 \\
& 8,6 \\
& 9,35\end{aligned}$ & $A / \Delta v=\begin{array}{r}8,3 \\
9,9 \\
13,9 \\
13,6\end{array}$ \\
\hline PAS DE LISSAGE & $\begin{array}{l}\text { Dist. GAUS. No } 1 \\
(N=9, T=500)\end{array}$ & $\begin{array}{c}\text { DisT. GAUS. No } 2 \\
(N=17, T=500)\end{array}$ \\
\hline $\begin{array}{r}L=10 \\
17 \\
23 \\
29\end{array}$ & $A / \Delta v=\begin{array}{r}7,6 \\
10,7 \\
11,2 \\
12,7\end{array}$ & $A / \Delta v=\begin{array}{r}8,3 \\
9,8 \\
11,5 \\
8,9\end{array}$ \\
\hline
\end{tabular}

\section{II}

La «période» des grandes ondulations lentes de vitesse en fonction du temps n'est théoriquement autre que l'inter- valle moyen $Z$ entre les zéros successifs des fluctuations de vitesse. Or, les petites fluctuations d'amplitude moyenne $\Delta v$ introduisent toute une série de zéros parasites dont la prise en considération masquerait plus ou moins l'allure générale de la dérive des valeurs "lissées". Il faut donc essayer de se débarrasser de ces zéros parasites par un deuxième "lissage ", plus ou moins visuel, pour pouvoir apprécier plus exactement cette dérive. Mais l'intervalle $Z$ entre les zéros dépend considérablement du pas $L^{\prime}$ de ce deuxième "lissage ":

\begin{tabular}{|c|c|c|}
\hline $\begin{array}{l}\text { PAS DU } \\
\text { DEUXIĖME } \\
\text { LISSAGE }\end{array}$ & $\begin{array}{c}\text { DÉCIMALES DE } \pi \\
\quad(N=10) \\
(T=500, L=10)\end{array}$ & $\begin{array}{c}\text { DisT. GAUS.N" } 1 \\
\quad(N=9) \\
(T=500, L=10)\end{array}$ \\
\hline $\begin{array}{r}L^{\prime}=10 \\
13 \\
17 \\
47\end{array}$ & $\begin{array}{r}Z=19,91 \\
21,90 \\
29,20 \\
65,40\end{array}$ & $\begin{array}{r}Z=23,45 \\
24,72 \\
27,75 \\
54,86\end{array}$ \\
\hline
\end{tabular}

Il est, d'autre part, manifeste que l'intervalle moyen entre les zéros doit tendre vers l'infini, puisque la valeur "lissée " tend alors vers une constante.

La «période » des grandes ondulations lentes de vitesse en fonction du temps ne peut donc avoir qu'un caractère purement subjectif, contrairement à ce qui se passe pour les petites fluctuations de vitesse dont nous avons vu qu'elles comportent une période (ou, plus exactement, une pseudopériode moyenne $\Delta t$ ) objectivement bien définie. 
TABLEAU I. - DISTRIBUTION UNIFORME

Lissage par 10

\begin{tabular}{|c|c|c|c|c|c|c|c|c|c|c|c|c|c|c|c|c|c|c|c|}
\hline \multicolumn{2}{|c|}{1} & \multicolumn{2}{|c|}{2} & \multicolumn{2}{|c|}{3} & \multicolumn{2}{|c|}{4} & \multicolumn{2}{|c|}{5} & \multicolumn{2}{|c|}{6} & \multicolumn{2}{|c|}{7} & \multicolumn{2}{|c|}{8} & \multicolumn{2}{|c|}{9} & \multicolumn{2}{|c|}{10} \\
\hline 1 & & 8 & 47 & 5 & 61 & 2 & 53 & 8 & 55 & 4 & 61 & 4 & 54 & 10 & 50 & 4 & 57 & 6 & 47 \\
\hline 4 & & 3 & 47 & 8 & 60 & 10 & 55 & 2 & 53 & 6 & 61 & 8 & 59 & 5 & 45 & 4 & 57 & 4 & 47 \\
\hline I & & 2 & 41 & 2 & 59 & 8 & 62 & 1 & 52 & 10 & 65 & 1 & 55 & 5 & 49 & 2 & 50 & 8 & 51 \\
\hline 5 & & 7 & 44 & 10 & 60 & 9 & 65 & 4 & 55 & 9 & 70 & 1 & 47 & 5 & 45 & 8 & 55 & 2 & 47 \\
\hline 9 & & 9 & 47 & 9 & 60 & 9 & 70 & 8 & 62 & 5 & 68 & 1 & 44 & 9 & 51 & 8 & 53 & 3 & 49 \\
\hline 2 & & 5 & 50 & 7 & 64 & 8 & 68 & 10 & 65 & 5 & 63 & 7 & 41 & 6 & 49 & 1 & 51 & 3 & 50 \\
\hline 6 & & 10 & 54 & 4 & 61 & 6 & 68 & 8 & 63 & 10 & 64 & 4 & 37 & 4 & 48 & 10 & 53 & 7 & 49 \\
\hline 5 & & 2 & 52 & 9 & 65 & 2 & 68 & 6 & 63 & 5 & 66 & 5 & 41 & 4 & 50 & 9 & 61 & 8 & 53 \\
\hline 3 & & 8 & 57 & 4 & 68 & 8 & 68 & 5 & 61 & 8 & 66 & 10 & 49 & 6 & 55 & 7 & 59 & 6 & 52 \\
\hline 5 & 41 & 8 & 62 & 4 & 62 & 10 & 72 & 1 & 53 & 2 & 64 & 2 & 43 & 2 & 56 & 5 & 58 & 7 & 54 \\
\hline 8 & 48 & 4 & 58 & 5 & 62 & 3 & 73 & 3 & 48 & 2 & 62 & 8 & 47 & 2 & 48 & 6 & 60 & 8 & 56 \\
\hline 9 & 53 & 1 & 56 & 9 & 63 & 4 & 67 & 2 & 48 & 3 & 59 & 4 & 43 & 9 & 52 & 6 & 62 & 3 & 55 \\
\hline 7 & 59 & 9 & 63 & 2 & 63 & 8 & 67 & 8 & 55 & 1 & 50 & 1 & 43 & 4 & 51 & 5 & 65 & 1 & 48 \\
\hline 9 & 63 & 7 & 63 & 3 & 56 & 2 & 60 & 2 & 53 & 7 & 48 & 10 & 52 & 8 & 54 & 9 & 66 & 6 & 52 \\
\hline 3 & 57 & 1 & 55 & 10 & 57 & 5 & 56 & 3 & 48 & 2 & 45 & 2 & 53 & 9 & 54 & 3 & 61 & 5 & 54 \\
\hline 2 & 57 & 6 & 56 & 7 & 57 & 3 & 51 & 10 & 48 & 5 & 45 & 7 & 53 & 5 & 53 & 3 & 63 & 2 & 53 \\
\hline 3 & 54 & 9 & 55 & 8 & 61 & 4 & 49 & 6 & 46 & 3 & 38 & 10 & 59 & 4 & 53 & 4 & 57 & 7 & 53 \\
\hline 8 & 57 & 3 & 56 & 1 & 53 & 2 & 49 & 6 & 46 & 5 & 38 & 1 & 55 & 9 & 58 & 4 & 52 & 1 & 46 \\
\hline 4 & 58 & 9 & 57 & 6 & 55 & 1 & 42 & 4 & 45 & 9 & 39 & 9 & 54 & 3 & 55 & 6 & 51 & 2 & 42 \\
\hline 6 & 59 & 9 & 58 & 4 & 55 & 1 & 33 & 7 & 51 & 4 & 41 & 3 & 55 & 10 & 63 & 1 & 47 & 10 & 45 \\
\hline 2 & 53 & 3 & 57 & 10 & 60 & 7 & 37 & 10 & 58 & 10 & 49 & 8 & 55 & 3 & 64 & 2 & 43 & 1 & 38 \\
\hline 6 & 50 & 7 & 63 & 6 & 57 & 10 & 43 & 9 & 65 & 8 & 54 & 5 & 56 & 8 & 63 & 8 & 45 & 9 & 44 \\
\hline 4 & 47 & 5 & 59 & 2 & 57 & 6 & 41 & 3 & 60 & 1 & 54 & 2 & 57 & 1 & 60 & 4 & 44 & 10 & 53 \\
\hline 3 & 41 & 1 & 53 & 8 & 62 & 7 & 46 & 8 & 66 & 2 & 49 & 1 & 48 & 9 & 61 & 7 & 42 & 9 & 56 \\
\hline 3 & 41 & 10 & 62 & 6 & 58 & 9 & 50 & 4 & 67 & 8 & 55 & 1 & 47 & 6 & 58 & 5 & 44 & 1 & 52 \\
\hline \multicolumn{2}{|c|}{11} & \multicolumn{2}{|c|}{12} & \multicolumn{2}{|c|}{13} & & & & & & & & & & & & & & \\
\hline 4 & 54 & 6 & 49 & 7 & 40 & 2 & 52 & 7 & 43 & 4 & 59 & 3 & 44 & 6 & 54 & 10 & 53 & 5 & 55 \\
\hline 5 & 52 & 6 & 47 & 2 & 40 & 10 & 54 & 8 & 50 & 6 & 60 & 3 & 43 & 1 & 54 & 7 & 59 & 2 & 51 \\
\hline 6 & 57 & 4 & 45 & 4 & 40 & 9 & 55 & 9 & 52 & 6 & 63 & 10 & 52 & 1 & 46 & 4 & 53 & 7 & 51 \\
\hline 4 & 59 & 8 & 52 & 5 & 36 & 2 & 56 & 2 & 53 & 5 & 58 & 5 & 52 & 7 & 48 & 4 & 52 & 2 & 50 \\
\hline 8 & 57 & 2 & 44 & 8 & 43 & 10 & 59 & 5 & 53 & 2 & 55 & 7 & 58 & 3 & 48 & 6 & 57 & 4 & 49 \\
\hline 5 & 61 & 1 & 41 & 7 & 46 & 9 & 64 & 9 & 59 & 1 & 52 & 2 & 59 & 8 & 46 & 2 & 58 & 8 & 56 \\
\hline 6 & 58 & 3 & 39 & 10 & 55 & 6 & 62 & 10 & 63 & 3 & 47 & 7 & 60 & 1 & 38 & 3 & 53 & 9 & 57 \\
\hline 6 & 54 & 3 & 38 & 10 & 63 & 2 & 56 & 3 & 62 & 8 & 47 & 10 & 60 & 9 & 45 & 7 & 55 & 1 & 50 \\
\hline 9 & 54 & 9 & 44 & 6 & 62 & 8 & 63 & 6 & 65 & 4 & 49 & 3 & 54 & 3 & 47 & 9 & 60 & 2 & 47 \\
\hline 2 & 55 & 3 & 45 & 6 & 65 & 2 & 60 & 10 & 69 & 1 & 40 & 6 & 56 & 2 & 41 & 9 & 61 & 2 & 42 \\
\hline 3 & 54 & 6 & 45 & 10 & 68 & 9 & 67 & 10 & 72 & 4 & 40 & 5 & 58 & 6 & 41 & 6 & 57 & 7 & 44 \\
\hline 4 & 53 & 10 & 49 & 6 & 72 & 2 & 59 & 1 & 65 & 6 & 40 & 7 & 62 & 1 & 41 & 2 & 52 & 9 & 51 \\
\hline 6 & 53 & 7 & 52 & 3 & 71 & 5 & 55 & 1 & 57 & 9 & 43 & 5 & 57 & 1 & 41 & 7 & 55 & 3 & 47 \\
\hline 10 & 59 & 2 & 46 & 1 & 67 & 4 & 57 & 3 & 58 & 5 & 43 & 9 & 61 & 7 & 41 & 4 & 55 & 8 & 53 \\
\hline 3 & 54 & 6 & 50 & 5 & 64 & 10 & 57 & 3 & 56 & 1 & 42 & 5 & 59 & 9 & 47 & 9 & 58 & 1 & 50 \\
\hline 4 & 53 & 10 & 59 & 5 & 62 & 9 & 57 & 10 & 57 & 9 & 50 & 9 & 66 & 3 & 42 & 5 & 61 & 8 & 50 \\
\hline 8 & 55 & 2 & 58 & 8 & 60 & 1 & 52 & 5 & 52 & 4 & 51 & 1 & 60 & 1 & 42 & 6 & 64 & 3 & 44 \\
\hline 6 & 55 & 4 & 59 & 8 & 58 & 7 & 57 & 3 & 52 & 1 & 44 & 9 & 59 & 10 & 43 & 7 & 64 & 10 & 53 \\
\hline 1 & 47 & 9 & 59 & 1 & 53 & 1 & 50 & 10 & 56 & 5 & 45 & 5 & 61 & 5 & 45 & 3 & 58 & 1 & 52 \\
\hline 10 & 55 & 1 & 57 & 7 & 54 & 5 & 53 & 5 & 51 & 1 & 45 & 3 & 58 & 1 & 44 & 5 & 54 & 1 & 51 \\
\hline 4 & 56 & 4 & 55 & 4 & 48 & 3 & 47 & 4 & 45 & 1 & 42 & 10 & 63 & 1 & 39 & 1 & 49 & 9 & 53 \\
\hline 5 & 57 & 1 & 46 & 8 & 50 & 6 & 51 & 8 & 52 & 6 & 42 & 9 & 65 & 8 & 46 & 8 & 55 & 4 & 48 \\
\hline 4 & 55 & 2 & 41 & 8 & 55 & 4 & 50 & 8 & 59 & 10 & 43 & 2 & 62 & 5 & 50 & 8 & 56 & 9 & 54 \\
\hline 3 & 48 & 7 & 46 & 1 & 55 & 3 & 49 & 2 & 58 & 9 & 47 & 1 & 54 & 4 & 47 & 5 & 57 & 1 & 47 \\
\hline 2 & 47 & 3 & 43 & 5 & 55 & 6 & 45 & 10 & 65 & 4 & 50 & 8 & 57 & 8 & 46 & 7 & 55 & 2 & 48 \\
\hline
\end{tabular}


TABLEAU II. - DISTRIBUTION GAUSSIENNE N ${ }^{\circ} 1$

Lissage par 10

\begin{tabular}{|c|c|c|c|c|c|c|c|c|c|c|c|c|c|c|c|c|c|c|c|}
\hline \multicolumn{2}{|c|}{1} & \multicolumn{2}{|c|}{2} & \multicolumn{2}{|c|}{3} & \multicolumn{2}{|c|}{4} & \multicolumn{2}{|c|}{5} & \multicolumn{2}{|c|}{6} & \multicolumn{2}{|c|}{7} & \multicolumn{2}{|c|}{8} & \multicolumn{2}{|c|}{9} & \multicolumn{2}{|c|}{10} \\
\hline 8 & & 5 & 56 & 8 & 55 & 6 & 40 & 4 & 41 & 4 & 37 & 5 & 47 & 4 & 49 & 3 & 50 & 4 & 50 \\
\hline 5 & & 9 & 60 & 7 & 54 & 5 & 43 & 9 & 45 & 5 & 41 & 6 & 49 & 7 & 51 & 4 & 48 & 6 & 50 \\
\hline 4 & & 5 & 60 & 4 & 54 & 4 & 41 & 1 & 42 & 3 & 42 & 4 & 44 & 4 & 49 & 3 & 50 & 6 & 49 \\
\hline 7 & & 6 & 59 & 4 & 53 & 8 & 47 & 6 & 43 & 4 & 40 & 5 & 45 & 4 & 48 & 4 & 47 & 7 & 51 \\
\hline 3 & & 5 & 59 & 4 & 51 & 3 & 47 & 3 & 40 & 7 & 43 & 4 & 44 & 6 & 49 & 7 & 49 & 5 & 51 \\
\hline 5 & & 7 & 57 & 3 & 52 & 5 & 45 & 6 & 41 & 1 & 41 & 4 & 44 & 4 & 50 & 5 & 48 & 6 & 52 \\
\hline 5 & & 6 & 59 & 6 & 52 & 4 & 45 & 5 & 43 & 6 & 42 & 7 & 47 & 6 & 54 & 9 & 54 & 5 & 50 \\
\hline 7 & & 4 & 56 & 4 & 50 & 4 & 45 & 3 & 43 & 6 & 43 & 6 & 47 & 5 & 53 & 2 & 48 & 4 & 51 \\
\hline 2 & & 3 & 54 & 4 & 49 & 6 & 49 & 4 & 46 & 5 & 46 & 5 & 48 & 7 & 53 & 8 & 54 & 5 & 51 \\
\hline 6 & 52 & 4 & 54 & 8 & 52 & 4 & 49 & 5 & 46 & 5 & 46 & 7 & 53 & 5 & 52 & 6 & 51 & 7 & 55 \\
\hline 1 & 45 & 6 & 55 & 3 & 47 & 2 & 45 & 7 & 49 & 7 & 49 & 3 & 51 & 5 & 53 & 4 & 52 & 7 & 58 \\
\hline 8 & 48 & 5 & 51 & 7 & 47 & 4 & 44 & 5 & 45 & 7 & 51 & 6 & 51 & 5 & 51 & 4 & 52 & 4 & 56 \\
\hline 4 & 48 & 4 & 50 & 8 & 51 & 2 & 42 & 6 & 50 & 6 & 54 & 4 & 51 & 6 & 53 & 5 & 54 & 4 & 54 \\
\hline 7 & 48 & 5 & 49 & 7 & 54 & 4 & 38 & 3 & 47 & 5 & 55 & 6 & 52 & 4 & 53 & 4 & 54 & 3 & 50 \\
\hline 8 & 53 & 4 & 48 & 5 & 55 & 5 & 40 & 2 & 46 & 3 & 51 & 6 & 54 & 5 & 52 & 7 & 54 & 7 & 52 \\
\hline 6 & 54 & 4 & 45 & 4 & 56 & 3 & 38 & 4 & 44 & 5 & 55 & 3 & 53 & 5 & 53 & 3 & 52 & 7 & 53 \\
\hline 5 & 54 & 8 & 47 & 2 & 52 & 5 & 39 & 1 & 40 & 4 & 53 & 5 & 51 & 6 & 53 & 6 & 49 & 8 & 56 \\
\hline 5 & 52 & 4 & 47 & 6 & 54 & 4 & 39 & 2 & 39 & 9 & 56 & 6 & 51 & 1 & 49 & 7 & 54 & 6 & 58 \\
\hline 7 & 57 & 5 & 49 & 2 & 52 & 5 & 38 & 6 & 41 & 4 & 55 & 5 & 51 & 7 & 49 & 5 & 51 & 5 & 58 \\
\hline 5 & 56 & 6 & 51 & 3 & 47 & 6 & 40 & 4 & 40 & 5 & 55 & 5 & 49 & 5 & 49 & 5 & 50 & 7 & 58 \\
\hline 9 & 64 & 2 & 47 & 7 & 51 & 5 & 43 & 3 & 36 & 4 & 52 & 3 & 49 & 6 & 50 & 5 & 51 & 4 & 55 \\
\hline 4 & 60 & 6 & 48 & 4 & 48 & 3 & 42 & 5 & 36 & 4 & 49 & 2 & 45 & 3 & 48 & 7 & 54 & 6 & 57 \\
\hline 7 & 63 & 6 & 50 & 4 & 44 & 3 & 43 & 5 & 35 & 6 & 49 & 6 & 47 & 8 & 50 & 3 & 52 & 3 & 56 \\
\hline 5 & 61 & 5 & 50 & 2 & 39 & 1 & 40 & 2 & 34 & 4 & 48 & 7 & 48 & 2 & 48 & 5 & 53 & 6 & 59 \\
\hline 4 & 57 & 5 & 51 & 4 & 38 & 5 & 40 & 5 & 37 & 2 & 47 & 6 & 48 & 9 & 52 & 3 & 49 & 6 & 58 \\
\hline \multicolumn{2}{|c|}{11} & \multicolumn{2}{|c|}{12} & \multicolumn{2}{|c|}{13} & & & & & & & & & & & & & & \\
\hline 4 & 55 & 7 & 55 & 6 & 55 & 3 & 50 & 4 & 50 & 6 & 49 & 6 & 58 & 3 & 55 & 5 & 50 & 5 & 63 \\
\hline 6 & 53 & 4 & 53 & 4 & 56 & 4 & 51 & 5 & 49 & 5 & 51 & 6 & 61 & 7 & 56 & 5 & 49 & 6 & 65 \\
\hline 5 & 52 & 9 & 57 & 7 & 57 & 7 & 53 & 5 & 53 & 6 & 53 & 3 & 59 & 5 & 53 & 6 & 52 & 4 & 62 \\
\hline 4 & 51 & 7 & 57 & 7 & 60 & 6 & 55 & 8 & 55 & 2 & 52 & 4 & 58 & 4 & 53 & 4 & 47 & 5 & 61 \\
\hline 7 & 51 & 5 & 56 & 4 & 60 & 5 & 54 & 8 & 57 & 8 & 53 & 7 & 57 & 5 & 53 & 5 & 48 & 3 & 59 \\
\hline 5 & 52 & 5 & 58 & 3 & 56 & 3 & 51 & 4 & 54 & 5 & 51 & 3 & 53 & 4 & 51 & 6 & 52 & 6 & 56 \\
\hline 8 & 54 & 6 & 58 & 5 & 55 & 6 & 52 & 5 & 52 & 6 & 54 & 5 & 52 & 4 & 50 & 5 & 50 & 2 & 51 \\
\hline 3 & 54 & 8 & 61 & 5 & 54 & 4 & 50 & 4 & 53 & 6 & 54 & 7 & 52 & 2 & 46 & 6 & 51 & 6 & 49 \\
\hline 6 & 54 & 4 & 60 & 6 & 53 & 6 & 50 & 4 & 52 & 3 & 52 & 4 & 49 & 6 & 47 & 6 & 53 & 5 & 48 \\
\hline 7 & 55 & 3 & 58 & 5 & 52 & 5 & 49 & 3 & 50 & 5 & 52 & 4 & 49 & 6 & 46 & 7 & 55 & 5 & 47 \\
\hline 7 & 58 & 4 & 55 & 8 & 54 & 5 & 51 & 6 & 52 & 5 & 51 & 3 & 46 & 6 & 49 & 8 & 58 & 5 & 47 \\
\hline 5 & 57 & 4 & 55 & 7 & 57 & 4 & 51 & 3 & 50 & 9 & 55 & 6 & 46 & 2 & 44 & 9 & 62 & 5 & 46 \\
\hline 8 & 60 & 6 & 52 & 4 & 54 & 3 & 47 & 4 & 49 & 5 & 54 & 5 & 48 & 3 & 42 & 8 & 64 & 4 & 46 \\
\hline 4 & 60 & 6 & 51 & 5 & 52 & 4 & 45 & 4 & 45 & 5 & 57 & 4 & 48 & 4 & 42 & 2 & 62 & 6 & 47 \\
\hline 5 & 58 & 4 & 50 & 7 & 55 & 6 & 46 & 5 & 42 & 5 & 54 & 6 & 47 & 5 & 42 & 3 & 60 & 3 & 47 \\
\hline 4 & 57 & 9 & 54 & 4 & 56 & 3 & 46 & 4 & 42 & 6 & 55 & 5 & 49 & 5 & 43 & 5 & 59 & 3 & 44 \\
\hline 6 & 55 & 3 & 51 & 3 & 54 & 6 & 46 & 3 & 40 & 3 & 52 & 6 & 50 & 6 & 45 & 4 & 58 & 3 & 45 \\
\hline 5 & 57 & 6 & 49 & 5 & 54 & 1 & 43 & 4 & 40 & 5 & 51 & 8 & 51 & 3 & 46 & 7 & 59 & 6 & 45 \\
\hline 7 & 58 & 4 & 49 & 4 & 52 & 6 & 43 & 3 & 39 & 5 & 53 & 4 & 51 & 9 & 49 & 6 & 59 & 5 & 45 \\
\hline 6 & 57 & 4 & 50 & 6 & 53 & 6 & 44 & 7 & 43 & 8 & 56 & 5 & 52 & 4 & 47 & 5 & 57 & 3 & 43 \\
\hline 3 & 53 & 7 & 53 & 6 & 51 & 7 & 46 & 7 & 44 & 7 & 58 & 6 & 55 & 2 & 43 & 9 & 58 & 6 & 44 \\
\hline 6 & 54 & 6 & 55 & 5 & 49 & 7 & 49 & 3 & 44 & 6 & 55 & 5 & 54 & 7 & 48 & 7 & 56 & 4 & 43 \\
\hline 5 & 51 & 6 & 55 & 6 & 51 & 3 & 49 & 6 & 46 & 7 & 57 & 6 & 55 & 5 & 50 & 8 & 56 & 5 & 44 \\
\hline 5 & 52 & 7 & 56 & 6 & 52 & 5 & 50 & 5 & 47 & 7 & 59 & 5 & 56 & 4 & 50 & 6 & 60 & 4 & 42 \\
\hline 5 & 52 & 6 & 58 & 6 & 51 & 5 & 49 & 5 & 47 & 4 & 58 & 7 & 57 & 5 & 50 & 6 & 63 & 6 & 45 \\
\hline
\end{tabular}

